\title{
A LERAY-SCHAUDER ALTERNATIVE FOR WEAKLY-STRONGLY SEQUENTIALLY CONTINUOUS WEAKLY COMPACT MAPS
}

RAVI P. AGARWAL, DONAL O’REGAN, AND XINZHI LIU

Received 23 June 2004 and in revised form 17 November 2004

A new applicable Leray-Schauder alternative is presented for weakly-strongly sequentially continuous maps. This result is then used to establish a general existence principle for operator equations.

\section{Introduction}

This paper presents new fixed point results for weakly sequentially upper semicontinuous maps defined on locally convex Hausdorff topological spaces which are angelic when furnished with the weak topology. Moreover, we establish an applicable Leray-Schauder alternative (Theorem 2.12) for a certain subclass of these maps. Our alternative combines the advantages of the strong topology (i.e., the sets are open in the strong topology) with the advantages of the weak topology (i.e., the maps are weakly-strongly sequentially continuous and weakly compact). In Section 3, we illustrate how easily Theorem 2.12 can be applied in practice.

Finally, we recall the following definition from the literature [9].

Definition 1.1. A Hausdorff topological space $X$ is said to be angelic if for every relatively countably compact set $C \subseteq X$, the following hold:

(i) $C$ is relatively compact,

(ii) for each $x \in \bar{C}$, there exists a sequence $\left\{x_{n}\right\}_{n \geq 1} \subseteq C$ such that $x_{n} \rightarrow x$.

Remark 1.2. All metrizable locally convex spaces equipped with the weak topology are angelic (see the Eberlein-Šmulian theorem).

\section{Fixed point theory}

We begin with some fixed point results which will be needed to obtain our applicable nonlinear alternative of Leray-Schauder type (see Theorem 2.12).

TheOREM 2.1. Let E be a locally convex linear Hausdorff topological space which is angelic when furnished with the weak topology, and let $C$ be a weakly compact, convex subset of $E$. 
Then any weakly sequentially upper semicontinuous map $F: C \rightarrow K(C)$ has a fixed point (here $K(C)$ denotes the family of nonempty, convex, weakly compact subsets of $C$ ).

Remark 2.2. Recall $F: C \rightarrow K(C)$ is weakly sequentially upper semicontinuous if for any weakly closed set $A$ of $C, F^{-1}(A)$ is sequentially closed for the weak topology on $C$.

Notice that the proof of Theorem 2.1 is immediate from Himmelberg's fixed point theorem [10] and the next result.

Theorem 2.3. Let E be a locally convex linear Hausdorff topological space which is angelic when furnished with the weak topology, and let $D$ be a weakly compact subset of $E$. If $F$ : $D \rightarrow 2^{E}$ (here $2^{E}$ denote the family of nonempty subsets of $E$ ) is a weakly sequentially upper semicontinuous map, then $F: D \rightarrow K(E)$ is a weakly upper semicontinuous map.

Proof. Let $A$ be a weakly closed subset of $E$. We first show that $F^{-1}(A)$ is sequentially closed in $D$ (with respect to the strong topology). (Recall that a subset $M$ is sequentially closed in $E$ (with respect to the strong topology) if whenever $x_{n} \in M$ for $n \in \mathbb{N}=\{1,2, \ldots\}$ and $x_{n} \rightarrow x$ (strong topology), then $x \in M$.)

Let $y_{n} \in F^{-1}(A)$ and $y_{n} \rightarrow y$ (strong topology). Then $y_{n} \rightarrow y$ (i.e., $y_{n} \rightarrow y$ in $(E, w)$ ). Now since $F: D \rightarrow 2^{E}$ is weakly sequentially upper semicontinuous (i.e., $F^{-1}(A)$ is sequentially closed in $(E, w)$ ), we have $y \in F^{-1}(A)$. Consequently if $A$ is a weakly closed subset of $D$, then $F^{-1}(A)$ is sequentially closed in $E$ (of course also weakly sequentially closed).

Now since $D$ is weakly compact, we have that $\overline{F^{-1}(A)^{w}}$ is weakly compact. Let $x \in$ $\overline{F^{-1}(A)^{w}}$. Now since $E$ is angelic when furnished with the weak topology, there exists a sequence $x_{n} \in F^{-1}(A)$ with $x_{n} \rightarrow x$. Also since $F^{-1}(A)$ is weakly sequentially closed, we have $x \in F^{-1}(A)$. Thus $\overline{F^{-1}(A)^{w}}=F^{-1}(A)$, so $F^{-1}(A)$ is weakly closed. Thus $F: D \rightarrow 2^{E}$ is a weakly upper semicontinuous map.

Our next result replaces the weak compactness of the space $C$ with a weak compactness assumption on the operator $F$. We present a number of results (see also $[2,5,6,11,12]$ ).

Theorem 2.4. Let E be a locally convex linear Hausdorff topological space which is angelic when furnished with the weak topology, and suppose the Krein-Šmulian property holds, and let $C$ be a closed, convex subset of $E$. Then any weakly compact, weakly sequentially upper semicontinuous map $F: C \rightarrow K(C)$ has a fixed point.

Remark 2.5. The Krein-Šmulian property states that the closed convex hull of a weakly compact set is weakly compact.

Remark 2.6. If $E$ is a Banach space, then we know [7, page 434] that the Krein-Šmulian property holds. For other examples, see [8, page 553] and [9, page 82].

Proof. There exists a weakly compact subset $A$ of $C$ with $F(C) \subseteq A \subseteq C$. The KreinŠmulian property guarantees that $\overline{\mathrm{co}}(A)$ is weakly compact. Notice also that $F: \overline{\mathrm{co}}(A) \rightarrow$ $K(\overline{\mathrm{CO}}(A))$, so Theorem 2.1 guarantees that there exists $x \in \overline{\mathrm{co}}(A)$ with $x \in F(x)$.

Theorem 2.7. Let E be a locally convex linear Hausdorff topological space which is angelic when furnished with the weak topology, and let $C$ be a closed convex subset of $E$ with $x_{0} \in C$. 
Suppose $F: C \rightarrow K(C)$ is a weakly sequentially upper semicontinuous map with the following property holding:

$$
A \subseteq C, \quad A=\overline{\mathrm{co}}\left(\left\{x_{0}\right\} \cup F(A)\right) \quad \text { implies } A \text { is weakly compact. }
$$

Then $F$ has a fixed point.

Proof. Consider $\mathscr{F}$ the family of all closed convex subsets $\Omega$ of $C$ with $x_{0} \in \Omega$ and $F(x) \subseteq$ $\Omega$ for all $x \in \Omega$. Note that $\mathscr{F} \neq \varnothing$ since $C \in \mathscr{F}$. Let $C_{0}=\cap_{\Omega \in \mathscr{F}} \Omega$. The argument in [11] guarantees that

$$
C_{0}=\overline{\mathrm{CO}}\left(\left\{x_{0}\right\} \cup F\left(C_{0}\right)\right) .
$$

Now (2.1) guarantees that $C_{0}$ is weakly compact and notice that (2.2) implies $F\left(C_{0}\right) \subseteq$ $C_{0}$. Also $F: C_{0} \rightarrow K\left(C_{0}\right)$ is weakly sequentially upper semicontinuous so Theorem 2.1 guarantees the existence of an $x_{0} \in C_{0}$ with $x_{0} \in F x_{0}$.

THeOREM 2.8. Let E be a locally convex linear Hausdorff topological space which is angelic when furnished with the weak topology, and let $C$ be a closed convex subset of $E$ with $x_{0} \in C$. Suppose $F: C \rightarrow K(C)$ is a weakly sequentially upper semicontinuous map with the following properties holding:

$$
\begin{aligned}
& A \subseteq C, \quad A=\operatorname{co}\left(\left\{x_{0}\right\} \cup F(A)\right) \quad \text { implies } \overline{A^{w}} \text { is weakly compact, } \\
& F^{-1}\left(\overline{A^{w}}\right) \text { is weakly closed for any weakly compact subset } A \text { of } C .
\end{aligned}
$$

Then $F$ has a fixed point.

Proof. Let

$$
\begin{gathered}
D_{0}=\left\{x_{0}\right\}, \quad D_{n}=\operatorname{co}\left(\left\{x_{0}\right\} \cup F\left(D_{n-1}\right)\right) \quad \text { for } n \in\{1,2, \ldots\}, \\
D=\cup_{n=0}^{\infty} D_{n} .
\end{gathered}
$$

The argument in [2, page 918] guarantees that

$$
D=\operatorname{co}\left(\left\{x_{0}\right\} \cup F(D)\right),
$$

so (2.3) implies that $\overline{D^{w}}$ is weakly compact. Consider the map $F^{\star}: \overline{D^{w}} \rightarrow K\left(\overline{D^{w}}\right)$ given by

$$
F^{\star}(x)=F(x) \cap \overline{D^{w}} .
$$

We need of course to check that $F^{\star}(x) \neq \varnothing$ for each $x \in \overline{D^{w}}$. Notice that (2.6) implies that $F(D) \subseteq D \subseteq \overline{D^{w}}$ so $D \subseteq F^{-1}\left(\overline{D^{w}}\right)$. Also $F^{-1}\left(\overline{D^{w}}\right)$ is a weakly closed from (2.4) so $\overline{D^{w}} \subseteq F^{-1}\left(\overline{D^{w}}\right)$, that is, $F^{\star}(x) \neq \varnothing$ for each $x \in \overline{D^{w}}$.

Also notice that $F^{\star}: \overline{D^{w}} \rightarrow K\left(\overline{D^{w}}\right)$ is weakly sequentially upper semicontinuous (note that $\left(F^{\star}\right)^{-1}(A)=F^{-1}(A) \cap \overline{D^{w}}$ for any subset $A$ of $\left.\overline{D^{w}}\right)$. Theorem 2.1 implies that there exists $x \in \overline{D^{w}}$ with $x \in F^{\star}(x) \subseteq F(x)$.

THeOREM 2.9. Let E be a locally convex linear Hausdorff topological space which is angelic when furnished with the weak topology and suppose that the Krein-Šmulian property holds, 
and let $C$ be a closed convex subset of $E$ with $x_{0} \in C$. Suppose $F: C \rightarrow K(C)$ is a weakly sequentially upper semicontinuous map with (2.4) satisfied and also assume that the following properties hold:

$$
\begin{gathered}
A \subseteq C, \quad A=\operatorname{co}\left(\left\{x_{0}\right\} \cup F(A)\right) \quad \text { with } \overline{A^{w}}=\overline{Q^{w}} \text { and } \\
Q \subseteq \text { A countable, implies } \overline{A^{w}} \text { is weakly compact }
\end{gathered}
$$

and

for any relatively weakly compact subset $A$ of $E$,

there exists a countable set $B \subseteq A$ with $\overline{B^{w}}=\overline{A^{w}}$.

Then $F$ has a fixed point.

Proof. Let $D_{n}$ and $D$ be as in Theorem 2.8 and notice that (2.6) holds. We claim $D_{n}$ is relatively weakly compact for each $n \in\{0,1,2, \ldots\}$. The case $n=0$ is immediate. Suppose $D_{k}$ is relatively weakly compact for some $k \in\{0,1, \ldots\}$. Then Theorem 2.3 guarantees that $F$ : $\overline{D_{k}^{w}} \rightarrow K(E)$ is weakly upper semicontinuous so [4] guarantees that $F\left(\overline{D_{k}^{w}}\right)$ is weakly compact. Now since the Krein-Šmulian property holds, then $D_{k+1}$ is relatively weakly compact. Thus $D_{n}$ is relatively weakly compact for each $n \in\{0,1,2, \ldots\}$. Now (2.9) implies that there exists $C_{n} ; C_{n}$ countable with $C_{n} \subseteq D_{n}$ and $\overline{C_{n}^{w}}=\overline{D_{n}^{w}}$. Let $C=\cup_{n=0}^{\infty} C_{n}$. The argument in [2, page 922] guarantees that $\overline{C^{w}}=\overline{D^{w}}$. This (together) with (2.8) and (2.6) implies that $\overline{D^{w}}$ is weakly compact. Let $F^{\star}: \overline{D^{w}} \rightarrow K\left(\overline{D^{w}}\right)$ be given by $F^{\star}(x)=F(x) \cap \overline{D^{w}}$. Notice also that $F^{\star}: \overline{D^{w}} \rightarrow K\left(\overline{D^{w}}\right)$ is weakly sequentially upper semicontinuous so Theorem 2.1 implies that there exists $x \in \overline{D^{w}}$ with $x \in F^{\star}(x) \subseteq F(x)$.

In applications, it is difficult and sometimes impossible to construct a set $C$ so that $F$ takes $C$ back into $C$. As a result, it makes sense to discuss map $F: C \rightarrow K(E)$. We present three Leray-Schauder alternatives. Our first result is for weakly sequentially upper semicontinuous maps, whereas our second and third results are for completely continuous maps (to be defined later).

THeOREM 2.10. Let E be a locally convex linear Hausdorff topological space which is angelic when furnished with the weak topology and suppose the Krein-Šmulian property holds, and let $C$ be a closed convex subset of $E, U$ a weakly open subset of $C, 0 \in U$, and $\overline{U^{w}}$ weakly compact (here $\overline{U^{w}}$ denotes the weak closure of $U$ in $C$ ). Suppose $F: \overline{U^{w}} \rightarrow K(C)$ is a weakly sequentially upper semicontinuous map which satisfies the following property:

$$
x \notin \lambda F x \quad \text { for every } x \in \partial U, \lambda \in(0,1)
$$

here $\partial U$ denotes the weak boundary of $U$ in $C$. Then $F$ has a fixed point in $\overline{U^{w}}$.

Proof. Suppose $F$ does not have a fixed point in $\partial U$ (otherwise we are finished), so $x \notin$ $\lambda F x$ for every $x \in \partial U$ and $\lambda \in[0,1]$. Consider

$$
A=\left\{x \in \overline{U^{w}}: x \in t F(x) \text { for some } t \in[0,1]\right\} .
$$


Now $A \neq \varnothing$ since $0 \in U$ and Theorem 2.3 guarantees that $F: \overline{U^{w}} \rightarrow K(C)$ is weakly upper semicontinuous. Thus $A$ is weakly closed, and in fact weakly compact since $\overline{U^{w}}$ is weakly compact.

Also $A \cap \partial U=\varnothing$ so there exists (since $(E, w)$, the space $E$ endowed with the weak topology, is completely regular) a weakly continuous map $\mu: \overline{U^{w}} \rightarrow[0,1]$ with $\mu(\partial U)=0$ and $\mu(A)=1$. Let

$$
J(x)= \begin{cases}\mu(x) F(x), & x \in \overline{U^{w}}, \\ \{0\}, & x \in C \backslash \overline{U^{w}} .\end{cases}
$$

Clearly, $J: C \rightarrow K(C)$ is a weakly compact, weakly sequentially upper semicontinuous map. Theorem 2.4 guarantees that there exists $x \in C$ with $x \in J(x)$. Notice that $x \in U$ since $0 \in U$. As a result $x \in \mu(x) F(x)$, so $x \in A$. Thus $\mu(x)=1$ and so $x \in F(x)$.

Remark 2.11. Notice that the assumption that $\overline{U^{w}}$ is weakly compact can be removed in Theorem 2.10 if $F: \overline{U^{w}} \rightarrow K(C)$ is weakly upper semicontinuous.

In applications, it is extremely difficult to construct the weakly open set $U$ in Theorem 2.10. This motivated us to construct a Furi-Pera-type theorem in [3]. In this paper, we present a new approach to maps which arise naturally in applications. Of course we would like also to remove the weak compactness of the domain space in Theorem 2.10 and replace it with the map being weakly compact. Our next theorem establishes such a result for a certain subclass of weakly sequential maps. The theorem combines the advantages of the strong topology (the sets are open in the strong topology) with the advantages of the weak topology (the maps are weakly-strongly sequentially continuous and weakly compact). As a result, we get a new applicable (see Section 3) fixed point theorem. We present the result for single-valued maps.

THEOREM 2.12. Let E be a locally convex linear Hausdorff topological space which is angelic when furnished with the weak topology. Let $C$ be a closed convex subset of $E, U$ a convex subset of $C$, and $U$ an open (strong topology) subset of $E$ with $0 \in U$. Suppose $F: \bar{U} \rightarrow C$ is a weakly-strongly sequentially continuous map (i.e., $F: \bar{U} \rightarrow C$ is completely continuous, i.e., if $x_{n}, x \in \bar{U}$ with $x_{n} \rightarrow x$, then $F x_{n} \rightarrow F x$, i.e., for any closed set $A$ of $C$, we have that $F^{-1}(A)$ is weakly sequentially closed); here $\bar{U}$ denotes the closure of $U$ in $C$. In addition, suppose either $\bar{U}$ is weakly compact or $F: \bar{U} \rightarrow C$ is weakly compact with the Krein-Šmulian property holding. Also assume that

$$
x \neq \lambda F x \quad \text { for } x \in \partial_{C} U, \lambda \in(0,1)
$$

here $\partial_{C} U$ denotes the boundary (strong topology) of $U$ in $C$. Then $F$ has a fixed point in $\bar{U}$. Remark 2.13. Note that int ${ }_{C} U=U$ (interior in the strong topology) since $U$ is open in $C$ so as a result, $\partial_{C} U=\partial_{E} U$; here $\partial_{E} U$ denotes the boundary of $U$ in $E$.

Proof. Let $\mu$ be the Minkowski functional on $\bar{U}$ and let $r: E \rightarrow \bar{U}$ be given by

$$
r(x)=\frac{x}{\max \{1, \mu(x)\}} \quad \text { for } x \in E .
$$


Note that $r: E \rightarrow \bar{U}$ is continuous. Also since $F: \bar{U} \rightarrow C$ is weakly-strongly sequentially continuous, we have immediately that $r F: \bar{U} \rightarrow \bar{U}$ is weakly sequentially continuous. Notice also that $r F: \bar{U} \rightarrow \bar{U}$ is a weakly compact map if $F: \bar{U} \rightarrow C$ is weakly compact; note that $\overline{F(\bar{U})^{w}}$ is weakly compact so the weak compactness of $r F$ follows from

$$
r\left(\overline{F(\bar{U})^{w}}\right) \subseteq \overline{\mathrm{CO}}\left(\{0\} \cup \overline{F(\bar{U})^{w}}\right)
$$

and the Krein-Šmulian property.

We apply Theorem 2.1 if $\bar{U}$ is weakly compact and Theorem 2.4 if $F: \bar{U} \rightarrow C$ is weakly compact. Thus there exists $x \in \bar{U}$ with $x=r F(x)$. Thus $x=r(y)$ with $y=F(x)$ and $x \in$ $\bar{U}=U \cup \partial U$ (note that $\operatorname{int}_{C} U=U$ since $U$ is also open in $C$ ). Now either $y \in \bar{U}$ or $y \notin \bar{U}$. If $y \in \bar{U}$, then $r(y)=y$ so $x=y=F(x)$, and we are finished. If $y \notin \bar{U}$, then $r(y)=$ $y / \mu(y)$ with $\mu(y)>1$. Then $x=\lambda y$ (i.e., $x=\lambda F(x)$ ) with $0<\lambda=1 / \mu(y)<1$; note that $x \in \partial_{C} U$ since $\mu(x)=\mu(\lambda y)=1$ (note that $\partial_{C} U=\partial_{E} U$ since int ${ }_{C} U=U$ ). This of course contradicts (2.13).

Remark 2.14. The argument above breaks down in the multivalued case (i.e., when $F$ : $\bar{U} \rightarrow K(C)$ ) since $r F: \bar{U} \rightarrow 2^{\bar{U}}$ but the values may not be convex. We will consider the multivalued case at a later stage using a different argument.

THeORem 2.15. Let E be a locally convex linear Hausdorff topological space which is angelic when furnished with the weak topology. Let $C$ be a closed convex subset of $E, U$ a convex subset of $C$, and $U$ an open (strong topology) subset of $E$ with $0 \in U$. Suppose $F: \bar{U} \rightarrow C$ is a weakly-strongly sequentially continuous map and assume that (2.13) and the following condition hold:

$$
D \subseteq \bar{U}, \quad D \subseteq \overline{\mathrm{CO}}(\{0\} \cup F(D)) \quad \text { implies } \overline{D^{w}} \text { is weakly compact }
$$

Then $F$ has a fixed point in $\bar{U}$.

Proof. Let $\mu$ and $r$ be as in Theorem 2.12 and note that $r F: \bar{U} \rightarrow \bar{U}$ is a weakly sequentially continuous map.

Let $A \subseteq \bar{U}$ with $A=\overline{c o}(\{0\} \cup r F(A))$. Now since $r F(A) \subseteq \operatorname{co}(\{0\} \cup F(A))$, we have

$$
A \subseteq \overline{\mathrm{CO}}(\{0\} \cup \operatorname{co}(\{0\} \cup F(A)))=\overline{\mathrm{CO}}(\{0\} \cup F(A))
$$

so (2.16) guarantees that $\overline{A^{w}}(=A)$ is weakly compact. Theorem 2.7 guarantees that there exists $x \in \bar{U}$ with $x=r F(x)$. Essentially, the same reasoning as in Theorem 2.12 completes the proof.

\section{Application}

In this section, we show how easily Theorem 2.12 can be applied in practice. We remark here that when one uses the standard Leray-Schauder (strong topology) alternative [1] in the literature, most of the work involves checking that the map is compact. This work is removed if one uses Theorem 2.12 (see Theorem 3.1). 
Consider the Dirichlet boundary value problem

$$
\begin{gathered}
y^{\prime \prime}+f\left(t, y, y^{\prime}\right)=0 \quad \text { a.e. on }[0,1], \\
y(0)=y(1)=0,
\end{gathered}
$$

where $f:[0,1] \times \mathbb{R}^{2} \rightarrow \mathbb{R}$ is an $L^{p}$-Carathéodory function with $p>1$. By this we mean

(i) $t \mapsto f(t, u, v)$ is measurable for all $(u, v) \in \mathbb{R}^{2}$,

(ii) $(u, v) \mapsto f(t, u, v)$ is continuous for a.e. $t \in[0,1]$,

(iii) for any $r>0$, there exists $h_{r} \in L^{p}[0,1]$ with $|f(t, u, v)| \leq h_{r}(t)$ for a.e. $t \in[0,1]$ and all $|u| \leq r$ and $|v| \leq r$.

By a solution to (3.1) we mean a function $y \in W^{2, p}[0,1]$ (i.e., $y^{\prime} \in A C[0,1]$ and $y^{\prime} \in$ $\left.L^{p}[0,1]\right)$, which satisfies the differential equation a.e. and $y(0)=y(1)=0$.

Define the operators

$$
H_{1}, H_{2}: L^{p}[0,1] \longrightarrow C[0,1] \subseteq L^{p}[0,1]
$$

by

$$
H_{1} u(t)=\int_{0}^{1} G(t, s) u(s) d s, \quad H_{2} u(t)=\int_{0}^{1} G_{t}(t, s) u(s) d s,
$$

where

$$
G(t, s)= \begin{cases}(t-1) s, & 0 \leq s \leq t \leq 1, \\ (s-1) t, & 0 \leq t \leq s \leq 1\end{cases}
$$

It is easy to see that solving (3.1) is equivalent to finding a solution $u \in L^{p}[0,1]$ to

$$
u=-f\left(t, H_{1}(u), H_{2}(u)\right) .
$$

Note that if $u$ is a solution of (3.5), then $y(t)=\int_{0}^{1} G(t, s) u(s) d s$ is a solution of (3.1), whereas if $w$ is a solution of (3.1), then $v=w^{\prime \prime}$ is a solution of (3.5).

Define an operator $F: L^{p}[0,1] \rightarrow L^{p}[0,1]$ by

$$
F u(t)=-f\left(t, H_{1}(u(t)), H_{2}(u(t))\right) .
$$

Consequently, solving (3.1) is equivalent to finding a fixed point $u \in L^{p}[0,1]$ to

$$
u=F u \text {. }
$$

Theorem 3.1. Let $f:[0,1] \times \mathbb{R}^{2} \rightarrow \mathbb{R}$ be an $L^{p}$-Carathéodory function with $p>1$ and suppose there is a constant $M_{0}$, independent of $\lambda$, with

$$
\left\|y^{\prime \prime}\right\|_{L^{p}}=\left(\int_{0}^{1}\left|y^{\prime \prime}(t)\right|^{p} d t\right)^{1 / p} \neq M_{0}
$$


8 Leray-Schauder alternative

for any solution $y$ to the problem

$$
\begin{gathered}
y^{\prime \prime}+\lambda f\left(t, y, y^{\prime}\right)=0 \quad \text { a.e. on }[0,1] \\
y(0)=y(1)=0
\end{gathered}
$$

for any $\lambda \in(0,1)$. Then (3.1) has at least one solution.

Proof. We will apply Theorem 2.12 with

$$
E=C=L^{p}[0,1], \quad U=\left\{u \in L^{p}[0,1]:\|u\|_{L^{p}}<M_{0}\right\} .
$$

Notice that $\bar{U}=\left\{u \in L^{p}[0,1]:\|u\|_{L^{p}} \leq M_{0}\right\}$ is closed and convex, so weakly closed. Moreover, $\bar{U}$ is weakly compact (recall that in a reflexive Banach space a subset is weakly compact if and only if it is closed in the weak topology and bounded in the norm topology). Also (3.8) guarantees that (2.13) holds. It remains to show that $F: \bar{U} \rightarrow L^{P}[0,1]$ is a weakly-strongly sequentially continuous map. Let $y_{n}, y \in \bar{U}$ with $y_{n} \rightarrow y$ in $L^{p}[0,1]$ (i.e., $\int_{0}^{1} y_{n} g d t \rightarrow \int_{0}^{1} y g d t$ for all $g \in L^{q}[0,1]$ with $\left.1 / p+1 / q=1\right)$. We must show that $F y_{n} \rightarrow F y$ in $L^{p}[0,1]$. Notice that

$$
\int_{0}^{1}\left|F y_{n}(t)-F y(t)\right|^{p} d t \leq \int_{0}^{1}\left|f\left(t, H_{1}\left(y_{n}\right), H_{2}\left(y_{n}\right)\right)-f\left(t, H_{1}(y), H_{2}(y)\right)\right|^{p} d t .
$$

If we show that

$$
\int_{0}^{1}\left|f\left(t, H_{1}\left(y_{n}\right), H_{2}\left(y_{n}\right)\right)-f\left(t, H_{1}(y), H_{2}(y)\right)\right|^{p} d t \longrightarrow 0 \quad \text { as } y_{n} \rightarrow y,
$$

then we are finished.

First we show, for each $t \in[0,1]$, that

$$
y_{n} \rightarrow y \quad \text { implies } H_{i}\left(y_{n}(t)\right) \longrightarrow H_{i}(y(t)) \text { for } i=1,2 .
$$

We prove (3.13) when $i=1$ (the case $i=2$ is similar). Fix $t \in[0,1]$. Then

$$
\left|H_{1}\left(y_{n}(t)\right)-H_{1}(y(t))\right|=\left|\int_{0}^{1} G(t, s)\left[y_{n}(s)-y(s)\right] d s\right| \longrightarrow 0
$$

as $y_{n} \rightarrow y$ since $G(t, \cdot) \in L^{q}[0,1]$ for fixed $t \in[0,1]$. Now (3.13) (together) with the fact that $f$ is an $L^{p}$-Carathéodory function gives

$$
y_{n} \rightarrow y \Longrightarrow f\left(t, H_{1}\left(y_{n}\right), H_{2}\left(y_{n}\right)\right) \longrightarrow f\left(t, H_{1}(y), H_{2}(y)\right) \quad \text { a.e. on }[0,1] \text {. }
$$

Also for $u \in \bar{U}$ and $t \in[0,1]$, we have

$$
\begin{aligned}
\left|H_{1}(u(t))\right| & =\left|\int_{0}^{1} G(t, s) u(s) d s\right| \\
& \leq\left(\int_{0}^{1}|u|^{p} d s\right)^{1 / p} \sup _{t \in[0,1]}\left(\int_{0}^{1}|G(t, s)|^{q} d s\right)^{1 / q} \\
& \leq M_{0} \sup _{t \in[0,1]}\left(\int_{0}^{1}|G(t, s)|^{q} d s\right)^{1 / q} .
\end{aligned}
$$


Thus there exists an $r>0$ with

$$
\left|H_{i}(u(t))\right| \leq r \quad \forall t \in[0,1], u \in \bar{U}, i=1,2 .
$$

Now (3.12) follows immediately from (3.15), (3.17), and the Lebesgue dominated convergence theorem.

We may now apply Theorem 2.12 to deduce that $F$ has a fixed point in $\bar{U}$.

The argument in Theorem 3.1 establishes the following existence principle for the operator equation

$$
u=T u,
$$

where $T: L^{p}[0,1] \rightarrow L^{p}[0,1]$ with $p>1$.

Theorem 3.2. Suppose there is a constant $M_{0}$, independent of $\lambda$, with

$$
\|y\|_{L^{p}} \neq M_{0}
$$

for any solution $y$ to the problem

$$
y=\lambda T y
$$

for any $\lambda \in(0,1)$. In addition, assume that $T: \bar{U} \rightarrow L^{p}[0,1]$ is a weakly-strongly sequentially continuous map; here $\bar{U}=\left\{u \in L^{p}[0,1]:\|u\|_{L^{p}} \leq M_{0}\right\}$. Then (3.18) has at least one solution in $\bar{U}$.

Remark 3.3. Of course there is an analog of Theorem 3.2 for the operator equation (3.18) where $T: E \rightarrow E$ with $E$ a reflexive Banach space (e.g., $E$ could be the Sobolev space $W^{k, p}\left([0,1], \mathbb{R}^{n}\right)$ with $k \geq 0$ and $\left.1<p<\infty\right)$.

\section{References}

[1] R. P. Agarwal, M. Meehan, and D. O'Regan, Fixed Point Theory and Applications, Cambridge Tracts in Mathematics, vol. 141, Cambridge University Press, Cambridge, 2001.

[2] R. P. Agarwal and D. O'Regan, Fixed-point theory for set valued mappings between topological vector spaces having sufficiently many linear functionals, Comput. Math. Appl. 41 (2001), no. 7-8, 917-928.

[3] - Fixed-point theory for weakly sequentially upper-semicontinuous maps with applications to differential inclusions, Nonlinear Oscil. 5 (2002), no. 3, 277-286.

[4] C. D. Aliprantis and K. C. Border, Infinite-Dimensional Analysis, Studies in Economic Theory, vol. 4, Springer-Verlag, Berlin, 1994.

[5] O. Arino, S. Gautier, and J.-P. Penot, A fixed point theorem for sequentially continuous mappings with application to ordinary differential equations, Funkcial. Ekvac. 27 (1984), no. 3, 273279.

[6] R. Bader, A topological fixed-point index theory for evolution inclusions, Z. Anal. Anwendungen 20 (2001), no. 1, 3-15.

[7] N. Dunford and J. T. Schwartz, Linear Operators. I. General Theory, Pure and Applied Mathematics, vol. 7, Interscience Publishers, New York, 1958.

[8] R. E. Edwards, Functional Analysis. Theory and Applications, Holt, Rinehart and Winston, New York, 1965. 


\section{Leray-Schauder alternative}

[9] K. Floret, Weakly Compact Sets, Lecture Notes in Mathematics, vol. 801, Springer, Berlin, 1980.

[10] C. J. Himmelberg, Fixed points of compact multifunctions, J. Math. Anal. Appl. 38 (1972), 205207.

[11] H. Mönch, Boundary value problems for nonlinear ordinary differential equations of second order in Banach spaces, Nonlinear Anal. 4 (1980), no. 5, 985-999.

[12] R. Precup, Fixed point theorems for decomposable multi-valued maps and applications, Z. Anal. Anwendungen 22 (2003), no. 4, 843-861.

Ravi P. Agarwal: Department of Mathematical Sciences, Florida Institute of Technology, Melbourne, FL 32901-6975, USA

E-mail address: agarwal@fit.edu

Donal O'Regan: Department of Mathematics, National University of Ireland, Galway, Ireland

E-mail address: donal.oregan@nuigalway.ie

Xinzhi Liu: Department of Applied Mathematics, University of Waterloo, ON, Canada N2L 3G1

E-mail address: xzliu@monotone.uwaterloo.ca 